\title{
Simplification of Galactic Dynamic equations
}

\author{
Ying-Qiu Gu* \\ School of Mathematical Science, Fudan University, Shanghai 200433, China
}

\begin{abstract}
Most fully developed galaxies have vivid spiral structure, but the formation and evolution of spiral structure is still a mystery that is not fully understood in astrophysics. We find that the currently used equations of galactic dynamics contain some unreasonable components. In this paper, the following three working assumptions are introduced to simplify the galactic structural equations. 1. In the research of large-scale structure, the retarded potential of the gravitational field should be taken into account. The propagating time of the gravitational field from center to border is longer than the revolution periods of the stars near the center of galaxy. Newton's gravitational potential is unreasonable for such case, and the weak field and low velocity approximation of Einstein's field equation should be adopted. 2. The stars in a fully developed galaxy should be zero-pressure and inviscid fluid, and the equation of motion is different from that of ordinary continuum mechanics. Stars move along geodesics. 3. The structure of the galaxy is only related to the total mass density distribution. The equation of state of dark halo is different from that of ordinary luminous interstellar matter, so their trajectories are also very different. Dark halo and ordinary matter in galaxy are automatically separated. The total mass density distribution can be presupposed according to the observation data, and then it can be determined by comparing the solution of the equations with the observed data.

These assumptions and treatments are supported by theory and observation. The variables of the equations of simplified galactic dynamics are separated from each other, and the equations are well-posed and can be solved according to a definite procedure. Therefore, this simplified dynamic equation system provides a more reasonable and practical framework for the further study of galactic structure, and can solve many practical problems. Besides, it is closely related to the study of dark energy and dark matter.
\end{abstract}

Keywords: galactic dynamics; spiral structure; bared spiral structure; density wave; gravitational retarded potential

PACS numbers: 98.52.Nr, 98.62.Hr, 98.62.Dm, 98.10.+z, 98.35.Df

*Electronic address: yqgu@fudan.edu.cn 


\section{INTRODUCTION}

Most fully developed galaxies have vivid spiral structures that have a profound effect on the In the past 70 years, a great deal of research has been done to reveal the essence of spiral structures. Although great progress has been made in understanding the mechanism of spiral arm formation, there are still many mysteries to be revealed. We don't even know whether the spiral is a longstanding phenomenon, or whether it is a transient and multiple regeneration phenomenon in the evolution of galaxies [1-3]. The prevailing view now is that the galactic spiral structure should be a quasi-stationary density wave[4]. According to the concept of density wave proposed by B. Lindblad, C. C. Lin and F. H. Shu analyzed and solved the quasi-stable solution of planetary fluid dynamics in gravitational field. But there are other ideas that the spiral structure is a short-lived but recurring spiral pattern formed by the vibration amplification in the rotating galaxy disk [5], or caused by external forces [6]. In the literatures [7, 8], by comparing the distant galaxies observed by the Hubble Space Telescope with those observed on the ground, the results suggest that the spiral structure in the range of $0<z<1$ in the universe may be a long-standing quasi-stationary pattern.

In density wave theory, Lin and Shu assume that the spiral structure is quasi-stationary in the form of waves on the disk of the galaxy. The key points of this assumption are as follows: 1 . The spiral galaxy has an invisible background gravitational field, which has a regular spiral structure, and the visible optical spiral arm is only the external manifestation of this spiral force field. 2 . The galactic spiral pattern is made up of flowing materials, which is not the fixed same materials on the spiral arm, but changes over time. 3. The entire spiral pattern is quasi-steady and rotates at constant angular velocity $\Omega$ like a rigid body. The above assumption is called the quasi-stable spiral structure assumption.

A spiral galaxy consists of at least three parts: disk, bulge and halo. Because of the large dispersion velocity of the two parts of the bulge and halo, it is not easy to produce density disturbance, so it is usually treated as rigid body, and its function is to form a part of the fundamental gravitational field. Only the galactic disk is part of the density wave. In terms of composition, most of the matter in the galactic disk is a star, but there is also a small amount of gas. Because of the great distance between stars, the average'collision' interval is several orders of magnitude larger than the age of the galaxy, so the collision can be omitted.

If the spiral arm of a spiral galaxy is made of fixed matter, and the matter near the center of the galaxy rotates faster than the matter at the edge of the galaxy, then the spiral arm becomes 
tighter and tighter. After only a few cycles, the arms become entangled and indistinguishable. Density wave theory has succeeded in avoiding this contradiction, with the arm matter not made up of fixed planets but of regions with higher star densities, similar to traffic jams on highways. For dynamic reasons, luminous matter such as a star moves slowly within the spiral arm, lingering for more time and gathering together, but the stars outside the spiral arm move fast and have lower densities.

Despite the great success of density wave theory, there are still some defects and problems. (1) nonlinear effects; (2) analysis of the open arm and barred spiral system; (3) detailed study of the near-central region; (4) dynamical classification of galaxies and so on. When using density wave theory to make galaxy arm pattern, for some cases, the rotary arm pattern is in good agreement with the actual one. But some other galaxies are not the case, and the barred spiral structure is not clearly explained. The better coincident part is mainly on the outer side of the spiral galaxy, but the results are often far from the actual observations when approaching the center. In theory, the perturbation gravitational potential formed by the density wave should be consistent with the response of the corresponding physical disk, but why would there be a big deviation between the two? Is the theory itself existing the question or caused by the other factors needs a further discussion.

The orbit of a star in a non-central potential field is usually a non-closed and complicated spatial curve, which is closely related to the initial velocity and its direction[1]. Therefore, it is more reasonable to take the star system in a galaxy as fluid rather than mass points. Although the dynamics of both models are essentially the same Newtonian mechanics, the initial and boundary conditions are set differently. In the case of fluid, the streamlines are consistent fields, which are a natural result of stars generating from nebula.

W. Dehnen and J. Binney fitted the mass distribution within the galaxy with the observed data(such as the rotation curve and the Oort constant [9]), but found that the mass distribution of the fitting was uncertain. In $[10,11]$, the authors numerically simulated the two-dimensional stellar fluid dynamics embedded in a planar galactic disk in dark matter halo and solved the Boltzmann equation to the second-order moment[1]. The references $[12,13]$ propose a kinetic approach to explain the formation of spirals and rings in galaxy clusters based on spiral arms, rings, and pseudo-rings driven near the unstable equilibrium point of a given rotating barred potential, and so on, associated with the invariant manifold composed of periodic orbitals around the equilibrium point. By adjusting the kinetic parameters of the main galaxy, the spiral and ringed structures are obtained. 
In view of the difficulties in analyzing and solving the existing dynamics model of galaxy structure, this paper will simplify the dynamic equations of density wave model according to the observation and reasonable assumptions. In simplified dynamical equations, the physical variables are separated from each other, which bring great convenience to analysis and solution. The equations can be solved by fixed procedure, and give a clearer explanation for the spiral and barred structure of galaxies. Thus, this simplified system of equations provides a more reasonable and practical framework for further study of the galactic structure, which can solve many practical problems, and is closely related to the study of dark matter and dark energy.

\section{SIMPLIFICATION OF GALACTIC DYNAMIC EQUATION}

In the current theoretical analysis, the commonly used galactic dynamics is similar to the following one[14]

$$
\begin{aligned}
& \nabla^{2} \Phi=\kappa \rho, \\
& \left(\partial_{t}+\vec{V} \cdot \nabla\right) \vec{V}+2 \Omega \times \vec{V}=-\nabla \Phi-\frac{1}{\rho} \nabla P, \\
& \partial_{t} P+\nabla \cdot(P \vec{V})=-P(\gamma-1) \nabla \cdot \vec{V}-\Lambda, \\
& \partial_{t} \rho+\nabla \cdot(\rho \vec{V})=0,
\end{aligned}
$$

in which $\kappa=4 \pi G, \Omega$ is the angular speed of the galactic disk, $\gamma$ adiabatic index, $\Lambda$ the cooling term reflecting the gravitational disturbance. Some more complicated models also introduce viscous term.

Let's analyze several problems in the dynamic equations (2.4). 1. The Einstein's field equation of gravity is essentially a wave equation. The diameters of galaxies are over 100,000 light-years, so the effect of retarded potential of gravity should be taken into account in solving the dynamic equations in such cases. The stars near the center of a galaxy turn around the center several times before the change of the corresponding gravitational field reaches the edge of the galaxy. Whereas the Newton's gravity is an action at a distance and ignores this time delay. So there is a hidden danger to use (2.1) to calculate the gravitational field in such an occasion, and the relativistic effect must be considered in the large-scale structure. The weak field and low velocity approximation of the Einstein's field equation is as follows[15]

$$
\partial_{\alpha} \partial^{\alpha} \Phi=-\kappa \rho
$$

in which $\rho$ is the total equivalent mass density. 
2. In the early stages of galaxy formation, luminous matter consists of hot plasma. The evolution process is complex at this time, and the dynamics should indeed take into account the influence of pressure, temperature, viscosity, and even magneto fluid effect. But as galaxies mature, luminous matter forms stars, and the motion of the stellar system becomes more and more orderly, which is similar to what happened in the solar system. The stars and ordinary luminous matter in the galaxy are mainly moving along geodesics, and these materials form zero-pressure and non-viscous fluid, so the energy-momentum tensor of the fluid should be as follows

$$
T_{s}^{\mu \nu}=\rho_{s} U^{\mu} U^{\nu}
$$

where $\rho_{s}$ is the comoving mass density of stars and ordinary luminous particles, $U^{\mu}$ is the 4 -vectors speed distribution of ordinary matter. The ordinary matter satisfies the energy conservation law $T_{s ; \nu}^{\mu \nu}=0$ independent of dark halo. In the form of continuity equations and equations of motion, we obtain the dynamic equations of the stellar system as

$$
U^{\mu} \partial_{\mu} \rho_{s}+\rho_{s} U_{; \mu}^{\mu}=0, \quad U^{\nu} U_{; \nu}^{\mu}=0
$$

For convenience, we take $c=1$ as unit of speed. The equations (2.2) and (2.3) derived from continuum theory and thermodynamics are not consistent with the actual conditions as mentioned above. The variables in such equations are strongly coupled, which brings great inconvenience and complexity to the analysis and solution of the equations.

3. The equation of state of the dark halo is different from that of ordinary luminous interstellar matter. For dark halo with self-action nonlinear potential, the nonlinear potential has a large proportion in the energy-momentum tension, and its equation of state have the following forms[15$18]$

$$
T^{\mu \nu}=\left(\rho_{\text {tot }}+P\right) \mathcal{U}^{\mu} \mathcal{U}^{\nu}+(W-P) g^{\mu \nu}
$$

where $W=W\left(\rho_{\text {tot }}\right)$ corresponds to the nonlinear potential of the dark particles, which has the effect of negative pressure, and causes the moving trajectories of the dark halo to deviate from geodesics. Thus the dark halo is automatically separated from ordinary matter during galaxy formation. So near the solar system, there are few traces of the dark matter that dominates galaxies. Dark halo consists of diffuse gases, and the energy conservation law corresponding to (2.8) is given by $T_{; \nu}^{\mu \nu}=0$, which is a complete $1+3$ dimensional nonlinear field equation. To analyze or solve such equation system is extremely difficult. However, the equation system (2.6) can be regarded as a planar dynamics in the domain that deviates slightly from the center of the galaxy, and that greatly 
simplifies the analysis and solving process for the equations. The galactic dynamics currently used confuses the two different kinds of concepts in (2.6) and (2.8). For example, in equations (2.1)(2.4), the mass density $\rho$ in each equation should be different concept. Therefore, the generality and effectiveness of the traditional galactic dynamic equations are quite weak, and it is difficult to get the right results in accordance with the actual situation.

Taking into account all above factors, we obtain the galactic dynamic equations in Minkowski space-time under weak field and low speed approximations[15]

$$
\begin{aligned}
\partial_{\alpha} \partial^{\alpha} \Phi & =-\kappa \rho, \\
\frac{d}{d t} \vec{V} & \equiv\left(\partial_{t}+\vec{V} \cdot \nabla\right) \vec{V}=-\nabla \Phi, \\
\frac{d}{d t} \rho_{s} & \equiv\left(\partial_{t}+\vec{V} \cdot \nabla\right) \rho_{s}=-\rho_{s} \nabla \cdot \vec{V},
\end{aligned}
$$

where $\vec{V}(t, \vec{x})$ is the velocity field of ordinary matter, and the total equivalent mass density is given by

$$
\rho \doteq \rho_{\text {tot }}-2 W+3 P
$$

In the above galactic dynamic equations, the variables $\left(\rho, \vec{V}, \rho_{s}\right)$ are relatively separated from each other, and the equations for $\Phi$ and $\rho_{s}$ are linear and easy to be solved. These features are significant advantages over traditional dynamical equations (2.1)-(2.4). If the total mass density $\rho$ is known, the gravitational potential of $\Phi(t, \vec{x})$ can be integrated, and the velocity field of $\vec{V}$ or stellar orbits can be obtained from (2.10)

$$
\frac{d^{2}}{d t^{2}} \vec{X}(t)=-\nabla \Phi(t, \vec{X})
$$

If the gravitational potential $\Phi$ is independent of time, the mechanical energy of each star is conserved. Integrating the above equation, we get

$$
\frac{1}{2} \vec{V}^{2}+\Phi(\vec{X})=E\left(\vec{X}_{0}\right)
$$

Thus, the inappropriate approximation in traditional galactic dynamics brings unnecessary complexity of the equations.

In theory, the complete dynamic equation should include the continuity equation and the dynamic equation of the dark halo. But these equations rely on the equations of state for dark matter and dark energy, which is now an unsolved puzzle. As the following analysis shows, the lack of this knowledge can be compensated by observational data. For example, by the rotational velocity curves of the stars, we can then in turn derive some important information about the total density 
distribution and spiral structure of the dark halo. In some sense, we study the inverse problem of literatures $[9,12,13]$.

\section{STRUCTURAL EQUATIONS OF NON-WARPED STATIONARY GALAXIES}

In the context of general relativity, the structure of galaxies depends on the distribution and properties of dark halo, which is unrealistic in the present situation[15, 16, 19]. Because the gravity in the galactic disk is very weak, except for the region near the center, the single gravitational potential $\Phi$ can describe the spiral structure precisely enough. We express equations in spherical coordinate system $(t, r, \theta, \phi)$. In this case, we have $g_{k l}=\operatorname{diag}\left(1, r^{2}, r^{2} \sin ^{2} \theta\right)$ and the non-zero Christoffel symbols

$$
\left\{\begin{array}{l}
\Gamma_{\theta \theta}^{r}=-r, \quad \Gamma_{\phi \phi}^{r}=-r \sin ^{2} \theta, \quad \Gamma_{r \theta}^{\theta}=\Gamma_{r \phi}^{\phi}=\frac{1}{r} \\
\Gamma_{\phi \phi}^{\theta}=-\sin \theta \cos \theta, \quad \Gamma_{\theta \phi}^{\phi}=\cot \theta
\end{array}\right.
$$

Via 3- $d$ tensor calculus, (2.9) and (2.10) becomes

$$
\begin{aligned}
c^{-2} \partial_{t}^{2} \Phi-g^{k l}\left(\partial_{k l}-\Gamma_{k l}^{m} \partial_{m}\right) \Phi & =-\kappa \rho \\
\partial_{t} V^{m}+V^{k}\left(\partial_{k} V^{m}+\Gamma_{k l}^{m} V^{l}\right) & =-g^{m l} \partial_{l} \Phi
\end{aligned}
$$

Substituting (3.1) into the above equations, we get the dynamic system in spherical coordinate system as

$$
\begin{array}{r}
c^{-2} \partial_{t}^{2} \Phi-\left[\partial_{r}^{2}+\frac{2}{r} \partial_{r}+\frac{1}{r^{2}}\left(\partial_{\theta}^{2}+\cot \theta \partial_{\theta}+\frac{1}{\sin ^{2} \theta} \partial_{\phi}^{2}\right)\right] \Phi+\kappa \rho=0, \\
\left(\partial_{t}+V_{r} \partial_{r}+V_{\theta} \partial_{\theta}+V_{\phi} \partial_{\phi}\right) V_{r}-r V_{\theta}^{2}-r \sin ^{2} \theta V_{\phi}^{2}+\partial_{r} \Phi=0, \\
\left(\partial_{t}+V_{r} \partial_{r}+V_{\theta} \partial_{\theta}+V_{\phi} \partial_{\phi}\right) V_{\theta}+\frac{2}{r} V_{r} V_{\theta}-\sin \theta \cos \theta V_{\phi}^{2}+\frac{\partial_{\theta} \Phi}{r^{2}}=0, \\
\left(\partial_{t}+V_{r} \partial_{r}+V_{\theta} \partial_{\theta}+V_{\phi} \partial_{\phi}\right) V_{\phi}+\frac{2}{r} V_{r} V_{\phi}+2 \cot \theta V_{\theta} V_{\phi}+\frac{\partial_{\phi} \Phi}{(r \sin \theta)^{2}}=0,
\end{array}
$$

where the velocity of the stars is defined in the form of a three-dimensional contra-variant vector

$$
V_{r} \equiv \frac{d}{d t} r(t), \quad V_{\theta} \equiv \frac{d}{d t} \theta(t), \quad V_{\phi} \equiv \frac{d}{d t} \phi(t)
$$

(3.5)-(3.7) is the Newton's second law for stellar motion.

For the stars moving in the galactic disk, we have

$$
\theta=\frac{\pi}{2}, \quad V_{\theta} \equiv 0
$$


In this case (3.6) holds automatically, so we get the two-dimensional equation of motion and continuity equation for stellar fluid in non-warped disk as $[3,10,15]$

$$
\begin{aligned}
\left(\partial_{t}+V_{r} \partial_{r}+V_{\phi} \partial_{\phi}\right) V_{r}-r V_{\phi}^{2}+\partial_{r} \Phi & =0 \\
\left(\partial_{t}+V_{r} \partial_{r}+V_{\phi} \partial_{\phi}\right) V_{\phi}+\frac{2}{r} V_{r} V_{\phi}+\frac{\partial_{\phi} \Phi}{r^{2}} & =0 \\
\left(\partial_{t}+V_{r} \partial_{r}+V_{\phi} \partial_{\phi}\right) \Sigma+\left(\partial_{r} V_{r}+\partial_{\phi} V_{\phi}+\frac{1}{r} V_{r}\right) \Sigma & =0
\end{aligned}
$$

where $\Sigma \geq 0$ is the mass surface density of stars in the disk.

For quasi-stationary spiral structures, the galactic structure moves as a whole at constant angular velocity $\Omega$ around the $z$-axis. Therefore, under the coordinate transformation $\varphi=\phi-\Omega t$, the solution of the structural equation in the new coordinate system $(t, r, \theta, \varphi)$ is static, that is, the solution is independent of time $t$. So we get the following structural equations for non-warped galaxies

$$
\begin{aligned}
{\left[\partial_{r}^{2}+\frac{2}{r} \partial_{r}+\frac{1}{r^{2}}\left(\partial_{\theta}^{2}+\cot \theta \partial_{\theta}+\frac{1}{\sin ^{2} \theta} \partial_{\varphi}^{2}\right)-\frac{\Omega^{2}}{c^{2}} \partial_{\varphi}^{2}\right] \Phi } & =\kappa \rho, \\
\left(V_{r} \partial_{r}+V_{\varphi} \partial_{\varphi}\right) V_{r}-r\left(V_{\varphi}+\Omega\right)^{2}+\partial_{r} \Phi & =0 \\
\left(V_{r} \partial_{r}+V_{\varphi} \partial_{\varphi}\right) V_{\varphi}+\frac{2}{r} V_{r}\left(V_{\varphi}+\Omega\right)+\frac{1}{r^{2}} \partial_{\varphi} \Phi & =0 \\
\left(V_{r} \partial_{r}+V_{\varphi} \partial_{\varphi}\right) \Sigma+\left(\partial_{r} V_{r}+\partial_{\varphi} V_{\varphi}+\frac{1}{r} V_{r}\right) \Sigma & =0 .
\end{aligned}
$$

The terms related with $\Omega$ in the above formulas have obvious physical significance, $r\left(V_{\varphi}+\Omega\right)^{2}$ is centrifugal force, and $\frac{2}{r} V_{r} \Omega$ Coriolis force. Because of the background gravitational force, (3.13)(3.15) is represented in spherical coordinate system, but (3.16) is described in polar coordinate system.

\section{SECOND-ORDER APPROXIMATION OF STATIONARY GALACTIC STRUCTURAL EQUATIONS}

The equations (3.13)-(3.16) can be solved according to the following procedure: At first, a reasonable total mass density $\rho$ is assumed, which can be expressed as series expansion of spherical harmonic function $Y_{l m}(\theta, \varphi)$. The distribution of the dark halo can be determined inversely by comparing the calculated solutions with the observed data. Second, for given $\rho,(3.13)$ is a linear equation with respect to $\Phi$, which can be easily solved with natural boundary conditions. Third, by (3.14) and (3.15), the velocity function can be represented by the trigonometric series of $\varphi$. Due to symmetry, the trigonometric series has only the terms with an integer multiple of a fixed 
base frequency, usually only even terms. The fourth, equation (3.16) for the surface mass density is a first-order linear equation, which is also easy to solve. In this way, the stellar distribution and movement are perfectly determined for non-warped stationary galaxies.

In what follows, we demonstrate the solving process of using the triangular series expansion according to this procedure. Consider the following second-order approximation of mass and gravitational potential distributions

$$
\begin{aligned}
\rho & =\rho_{0}+\left(\rho_{1}+\rho_{2} \cos 2 \varphi+\rho_{3} \sin 2 \varphi\right) \sin ^{2} \theta \\
\Phi & =\Phi_{0}+\left(\Phi_{1}+\Phi_{2} \cos 2 \varphi+\Phi_{3} \sin 2 \varphi\right) \sin ^{2} \theta
\end{aligned}
$$

in which all $\left(\rho_{n}, \Phi_{n}\right)$ are functions of $r$, and satisfy $\rho \geq 0$. The corresponding second order velocity of the stars in the disk is given by

$$
\begin{aligned}
& V_{r}=v_{1} \cos 2 \varphi+v_{2} \sin 2 \varphi, \quad V_{\theta}=0, \\
& V_{\varphi}=\omega_{0}+\omega_{1} \cos 2 \varphi+\omega_{2} \sin 2 \varphi
\end{aligned}
$$

in which all $\left(v_{n}, \omega_{n}\right)$ are functions of $r$.

Substituting (4.1) and (4.2) into (3.13), we get the equation of $\Phi_{n}$ as

$$
\begin{aligned}
\Phi_{0}^{\prime \prime}+\frac{2}{r} \Phi_{0}^{\prime}+\frac{4}{r^{2}} \Phi_{1} & =\kappa \rho_{0}, \\
\Phi_{1}^{\prime \prime}+\frac{2}{r} \Phi_{1}^{\prime}-\frac{6}{r^{2}} \Phi_{1} & =\kappa \rho_{1}, \\
\frac{4 \Omega^{2}}{c^{2}} \Phi_{k}+\Phi_{k}^{\prime \prime}+\frac{2}{r} \Phi_{k}^{\prime}-\frac{6}{r^{2}} \Phi_{k} & =\kappa \rho_{k}, \quad(k=2,3) .
\end{aligned}
$$

The solution is given by

$$
\begin{aligned}
\Phi_{1} & =-\frac{\kappa}{5}\left(\frac{1}{r^{3}} \int_{0}^{r} \rho_{1}(\chi) \chi^{4} d \chi+r^{2} \int_{r}^{\infty} \frac{\rho_{1}(\chi)}{\chi} d \chi\right) \\
\Phi_{0} & =-\frac{1}{r} \int_{0}^{r}\left(\chi^{2} \kappa \rho_{0}-4 \Phi_{1}\right) d \chi-\int_{r}^{\infty} \frac{1}{\chi}\left(\chi^{2} \kappa \rho_{0}-4 \Phi_{1}\right) d \chi \\
\Phi_{k} & =\frac{\kappa c^{5}}{32 \Omega^{5} r^{2}}\left(A(r) \int_{0}^{r} B(\chi) \rho_{k}(\chi) d \chi+B(r) \int_{r}^{\infty} A(\chi) \rho_{k}(\chi) d \chi\right)
\end{aligned}
$$

in which

$$
\begin{aligned}
& A(r)=\frac{6 \Omega}{c} \cos \left(\frac{2 \Omega r}{c}\right)+\frac{4 \Omega^{2} r^{2}-3 c^{2}}{r c^{2}} \sin \left(\frac{2 \Omega r}{c}\right), \\
& B(r)=\frac{4 \Omega^{2} r^{2}-3 c^{2}}{r c^{2}} \cos \left(\frac{2 \Omega r}{c}\right)-\frac{6 \Omega}{c} \sin \left(\frac{2 \Omega r}{c}\right) .
\end{aligned}
$$


Substituting (4.2)-(4.4) into (3.14) and (3.15), and restricting $\theta=\frac{1}{2} \pi$, we get

$$
\begin{aligned}
0= & \frac{d}{d r}\left[\Phi_{0}+\Phi_{1}+\frac{1}{4}\left(v_{1}^{2}+v_{2}^{2}\right)\right]-\frac{1}{2} R\left[2\left(\omega_{0}+\Omega\right)^{2}+\omega_{1}^{2}+\omega_{2}^{2}\right]+ \\
& \omega_{1} v_{2}-\omega_{2} v_{1}+\left[2 \omega_{0} v_{2}-2 r\left(\omega_{0}+\Omega\right) \omega_{1}+\Phi_{2}^{\prime}\right] \cos 2 \varphi- \\
& {\left[2 \omega_{0} v_{1}+2 r\left(\omega_{0}+\Omega\right) \omega_{2}-\Phi_{3}^{\prime}\right] \sin 2 \varphi+\cdots }
\end{aligned}
$$

as well as

$$
\begin{aligned}
0= & \frac{1}{2}\left[\left(r \omega_{1}^{\prime}+2 \omega_{1}\right) v_{1}+\left(r \omega_{2}^{\prime}+2 \omega_{2}\right) v_{2}\right]+ \\
& {\left[\left(r \omega_{0}^{\prime}+2 \omega_{0}+2 \Omega\right) v_{1}+2 r \omega_{0} \omega_{2}+\frac{2}{r} \Phi_{3}\right] \cos 2 \varphi+} \\
& {\left[\left(r \omega_{0}^{\prime}+2 \omega_{0}+2 \Omega\right) v_{2}-2 r \omega_{0} \omega_{1}-\frac{2}{r} \Phi_{2}\right] \sin 2 \varphi+\cdots }
\end{aligned}
$$

Let the coefficients of the terms $(\sin 2 \varphi, \cos 2 \varphi)$ in $(4.13)$ and (4.14) be 0 , we get

$$
\begin{aligned}
& v_{1}=-K\left(\omega_{0} r^{2} \Phi_{3}^{\prime}+2 r\left(\omega_{0}+\Omega\right) \Phi_{3}\right) \\
& v_{2}=K\left(\omega_{0} r^{2} \Phi_{2}^{\prime}+2 r\left(\omega_{0}+\Omega\right) \Phi_{2}\right) \\
& \omega_{1}=K\left[\left(\frac{1}{2} r^{2} \omega_{0}^{\prime}+r\left(\omega_{0}+\Omega\right)\right) \Phi_{2}^{\prime}+2 \omega_{0} \Phi_{2}\right] \\
& \omega_{2}=K\left[\left(\frac{1}{2} r^{2} \omega_{0}^{\prime}+r\left(\omega_{0}+\Omega\right)\right) \Phi_{3}^{\prime}+2 \omega_{0} \Phi_{3}\right] \\
& K \equiv\left[r^{2}\left(r\left(\omega_{0}+\Omega\right) \omega_{0}^{\prime}+2 \Omega\left(2 \omega_{0}+\Omega\right)\right)\right]^{-1}
\end{aligned}
$$

Substituting the above results into (4.14), that is, into the equation

$$
\left(r \partial_{r} \omega_{1}+2 \omega_{1}\right) v_{1}+\left(r \partial_{r} \omega_{2}+2 \omega_{2}\right) v_{2}=0
$$

we get a first-order linear ordinary differential equation for $\omega_{0}$

$$
\omega_{0}^{\prime}=\frac{\omega_{0} r}{2} \cdot \frac{\Phi_{3}^{\prime} \Phi_{2}^{\prime \prime}-\Phi_{2}^{\prime} \Phi_{3}^{\prime \prime}}{\Phi_{3} \Phi_{2}^{\prime}-\Phi_{2} \Phi_{3}^{\prime}}+\left(\omega_{0}+\Omega\right) \frac{\Phi_{3} \Phi_{2}^{\prime \prime}-\Phi_{2} \Phi_{3}^{\prime \prime}}{\Phi_{3} \Phi_{2}^{\prime}-\Phi_{2} \Phi_{3}^{\prime}}+\frac{1}{r}\left(3 \omega_{0}+\Omega\right)
$$

and another solution with no physical meaning $\omega_{0}^{\prime}=-\frac{2}{r}\left(\omega_{0}+\Omega\right)$. It is important to note that, while $\Phi_{2} \equiv 0$ or $\Phi_{3} \equiv 0, \omega_{0}$ cannot be determined by above equation(4.21). In this case (4.20) holds automatically. Substituting (4.15)-(4.19) into (4.13), i.e., substituting them into

$$
\frac{d}{d r}\left[\Phi_{0}+\Phi_{1}+\frac{1}{4}\left(v_{1}^{2}+v_{2}^{2}\right)\right]=\frac{1}{2} r\left[2\left(\omega_{0}+\Omega\right)^{2}+\omega_{1}^{2}+\omega_{2}^{2}\right]-\omega_{1} v_{2}+\omega_{2} v_{1},
$$

we get a constraint between the mass density distribution and $\Omega$, so $\Omega$ is determined by the total mass density. From (4.15)-(4.21) we learn that, the stellar speed $\vec{V}$ is completely determined by $\left(\rho_{2}, \rho_{3}, \Omega\right)$. After the velocity distribution is determined, the surface mass density $\Sigma(r, \varphi)$ of the stars can be solved from (3.16). At this point, the second-order approximation of galactic dynamics is completely solved. The above process only involves solving ordinary differential equations. 


\section{SIMPLE SOLUTIONS TO STATIONARY GALAXY STRUCTURE}

The calculations in the preceding section show that the structure of a stationary galaxy is determined by $\left(\rho_{2}, \rho_{3}, \Omega\right)$. If the total mass density distribution is known, it is easy to determine the structure of the galaxy according to the above procedure. However, it is difficult to directly determine the density distribution. The data observed is mainly the moving speeds of the stars. High-precision observation show that the stellar speeds in galaxies in a larger range is approximately equal. To illustrate the above solving procedure in detail and get some enlightening conclusions, we concretely solve two of the simplest examples. As a working assumption, we assume[20-22],

$$
\omega_{0}=\frac{v}{r}-\Omega, \quad\left(r \in\left[R_{0}, R_{1}\right]\right)
$$

where $v$ is a constant velocity with a typical value of $|v|=200 \sim 400 \mathrm{~km} / \mathrm{s},\left[R_{0}, R_{1}\right]$ is the effective domain of assumption (5.1). Usually we have $R_{0}=100 \sim 500 \mathrm{pc}$ and $R_{1}=10 \sim 60 \mathrm{kpc}$, which are about the radius of the bulge and the radius of the visible range respectively. No loss of generality, we can set $\left(\omega_{0}>0, v>0\right)$, otherwise we can make a transformation $\phi \rightarrow-\phi$ to get this condition. So we always have $v>r \Omega$ in the effective domain. As shown below, using the star's velocity curve $v=v(r)$ to calculate the total mass density is a good method to study the properties of dark halo[15, 23-26].

\section{A. Barred spiral galaxy}

We consider the simple case $\rho_{2} \neq 0, \rho_{3}=0$. In this case we have $P_{3}=0$, and $P_{2}(r)$ can be calculated by (4.10). By (4.15)-(4.19), we get

$$
\begin{array}{ll}
v_{1}=0, & v_{2}=-\frac{r(v-r \Omega) P_{2}^{\prime}+2 v P_{2}}{2(v-r \Omega)^{2}-v^{2}}, \\
\omega_{2}=0, & \omega_{1}=-\frac{r v P_{2}^{\prime}+4(v-r \Omega) P_{2}}{2 r\left[2(v-r \Omega)^{2}-v^{2}\right] .}
\end{array}
$$

The effective region of the above formulas is $r<\frac{(2-\sqrt{2}) v}{2 \Omega}$. Substituting (5.1)-(5.3) into (3.16), we get the surface density of stars as

$$
\Sigma=\frac{1}{\Sigma_{0}+C_{2} r v_{2} \cos (2 \varphi)}
$$

in which

$$
\Sigma_{0}=r v_{2} e^{-\int \frac{2 \omega_{1}}{v_{2}} d r}\left(C_{1}+2 C_{2} \int \frac{v-r \Omega}{r v_{2}} e^{\int^{r} \frac{2 \omega_{1}(\chi)}{v_{2}(\chi)} d \chi} d r\right)
$$


$\left(C_{1}, C_{2}\right)$ are integration constants.

The above results show the structural characteristics of a barred spiral galaxy. For given total mass density $\rho$ and boundary values, all the above formulas can be calculated concretely, but the analytical expression is a little complicated. If a restriction $v_{2}=0$ is added, that is $V_{r} \equiv 0$, then the stars move around circles, the results are relatively simple. In this case, by (5.2), we have

$$
\Phi_{2}=-\frac{q}{r^{2}}(v-r \Omega)^{2}, \quad \Phi_{3}=0
$$

in which $q \geq 0$ is integration constant. Substituting (5.6) into (4.15)-(4.19) we get

$$
V_{r}=0, \quad V_{\varphi}=\frac{1}{r^{3}}(v-r \Omega)\left(r^{2}+q \cos 2 \varphi\right)
$$

Again by (3.16), we get the surface density of stars

$$
\Sigma=\frac{\varrho(r)}{r^{2}+q \cos 2 \varphi}
$$

in which $\varrho(r)$ is determined by boundary condition. Substituting the above results into (4.22), we get a restriction between $\Omega$ and potential

$$
\Phi_{0}+\Phi_{1}=v^{2} \ln \left(\frac{r}{r_{0}}\right)-\frac{q^{2}}{24 r^{4}}\left(3 v^{2}-8 v r \Omega+6 r^{2} \Omega^{2}\right), \quad\left(r_{0}>0\right)
$$

In the case of $q=0$, the solution (5.6)-(5.9) is exact, which correspond to elliptical galaxy. But as long as the conditions deviate a little, the elliptical galaxy will evolve into a spiral one. The spiral structure should be an inevitable result for the evolution of ordinary galaxies.

\section{B. Spiral galaxy}

Now we calculate the following simple case,

$$
\Omega=0, \quad \omega_{0}=\frac{v}{r}, \quad \Phi_{2}=P^{-1} \cos (\xi r), \quad \Phi_{3}=P^{-1} \sin (\xi r)
$$

where $\xi>0$ is a constant, $P(r)$ is a function of $r$. Substituting (5.10) into (4.21), we get a linear differential eguation for $P(r)$

$$
P^{\prime \prime}-\frac{4}{r} P^{\prime}+\left(\xi^{2}+\frac{8}{r^{2}}\right) P=0
$$

The solution is given by

$$
P=\sqrt{r^{5}}\left[C_{1} J_{\alpha}(\xi r)+C_{2} J_{-\alpha}(\xi r)\right], \quad \alpha=\frac{1}{2} \sqrt{7}
$$


in which $\left(C_{1}, C_{2}\right)$ are constants, $\left(J_{\alpha}, J_{-\alpha}\right)$ is Bessel function of imaginary parameter

$$
J_{\nu}(x)=\left(\frac{x}{2}\right)^{\nu} \sum_{k=0}^{\infty} \frac{1}{k !} \frac{1}{\Gamma(\nu+k+1)}\left(\frac{x}{2}\right)^{2 k}, \quad(\nu= \pm \alpha) .
$$

Substituting the above relations into (4.15)-(4.19), we have

$$
\begin{aligned}
\Phi & =\Phi_{0}+\left[\Phi_{1}+P^{-1} \cos \alpha\right] \sin ^{2} \theta, \quad \alpha \equiv \xi r-2 \varphi \\
V_{r} & =\frac{1}{v P^{2}}\left[\xi r P \cos \alpha-\left(r P^{\prime}-2 P\right) \sin \alpha\right], \\
r V_{\varphi} & =\frac{1}{2 v P^{2}}\left[\left(r P^{\prime}-4 P\right) \cos \alpha+\xi r P \sin \alpha\right]+v, \\
\Sigma & =\frac{C_{3} P^{2}}{\xi r^{5}}\left(\left(r P^{\prime}-2 P\right) \cos \alpha+\xi r P \sin \alpha+2 r^{2} v^{2} \int \frac{P^{2}}{r^{3}} d r\right) .
\end{aligned}
$$

All of these variables are characterized by the structure of spiral galaxy, which are closer to the Archimedes spiral rather than the logarithmic one. Since the pitch angle of galaxy is approximately $10^{\circ} \sim 40^{\circ}[27]$, we have estimation $\xi=(2 \sim 11) R_{1}^{-1}$.

\section{CONCLUSION}

For the mature galaxies, three working hypotheses are introduced to simplify the dynamic equations: 1. To research the Large-scale structure of galaxy, we should consider the retarded potential of gravity, which takes longer time to propagate to border of galaxy than the revolution period of the stars near the center. The Newtonian gravitational potential is unreasonable in this case and should be replaced by wave equation, i.e., the weak field and low velocity approximation of Einstein's field equation (2.5).

2. The stellar system of mature galaxies should be zero-pressure and viscosity-free fluid with different equations of motion from ordinary continuum mechanics. The pressure of such system has no physical meaning and cannot be defined, and the introduction of pressure will only bring errors and troubles. The star moves along geodesic and the dynamic equation of stellar system should be (2.7).

3. The equation of state of dark halo is different from that of ordinary luminous interstellar matter, so their trajectories are very different, and then the dark halo is automatically separated from ordinary matter in galaxies. The structure of galaxies is only determined by the total mass density, which can be assumed beforehand based on observations. Then the total mass density can be determined by comparing the solution of structural equations with the observed data.

By these treatments, we find that the variables in the dynamical equations of galaxies are separated from each other. The equations are well-posed and can be solved according to a fixed 
procedure. Traditional equations are too complicated to be studied in depth. In fact, the basic objects of Nature are designed elaborately, and the corresponding equations are simple and symmetrical. If our research is trapped in complex computation and incomprehensible dilemma, this situation reflects that the model used has gone wrong with big probability. The dynamic equation system simplified above provides a more reasonable and practical framework for studying the structure of galaxies and is closely linked to the study of dark matter and dark energy.

[1] B. Binney, S. Tremaine, Galactic Dynamics (Ch.3, Ch.4, Ch.6), Princeton University Press, 1987

[2] N. Orlova, et al, Application of the global modal approach to the spiral galaxies, Astrophys. Space Sci. 284 (2003)739-742, arXiv:astro-ph/0211538

[3] S. E. Meidt, et al, Tests of the radial Tremaine-Weinberg Method, arXiv:0711.4104

[4] C. C. Lin, F. H. Shu, On the Spiral Structure of Disk Galaxies, Astrophys. J., 140, 646(1964)

[5] Goldreich P. , Lynden-Bell D., II. Spiral Arms as Sheared Gravitational Instabilities[J]. MNRAS, 1965, $130(2): 125-158$.

[6] W. H. Julian, A. Toomre, Non-Axisymmetric Responses of Differentially Rotating Disks of Stars[J]. Astrophys. J., 1966, 146(3):810.

[7] R. S. Somerville, et al, An Explanation for the Observed Weak Size Evolution of Disk Galaxies, Astrophys. J., 672(2), pp. 776-786(2008), arXiv:astro-ph/0612428

[8] Kendall S, Kennicutt R C, Clarke C , et al. Tracing spiral density waves in M81[J]. MNRAS, 2008, 387(3):1007-1020., arXiv:0804.2380v1

[9] W. Dehnen, J. Binney, Mass models of the Milky Way, MNRAS, 294, 429(1998), arXiv:astro$\mathrm{ph} / 9612059$

[10] E. I. Vorobyov, Ch. Theis, Boltzmann moment equation approach for the numerical study of anisotropic stellar disks, MNRAS, 373, 197-208(2006), arXiv:astro-ph/0609250

[11] E. I. Vorobyov, Ch. Theis, Shape and orientation of stellar velocity ellipsoids in spiral galaxies, Accepted by MNRAS, arXiv:0709.2768

[12] M. Romero-Gómez, et al, The formation of spiral arms and rings in barred galaxies, arXiv:0705.2958

[13] M. Romero-Gómez, The Formation of Spiral Arms and Rings in Barred galxies, arXiv:0801.0366

[14] A. Riols, H. Latter, Spiral density waves and vertical circulation in protoplanetary discs, MNRAS 000, 113 (2017) arXiv:1802.06620

[15] Y. Q. Gu, Stationary Spiral Structure and Collective Motion of the Stars in a Spiral Galaxy, Old Problems and New Horizons in World Physics (Ch.12), V. Krasnoholovets, V. Christianto, F. Smarandache, (Eds), Nova Science Publishers, New York, 2019, arXiv:0805.2828

[16] Y. Q. Gu, Functions of State for Spinor Gas in General Relativity, OALib. J. 4, e3953 (2017). arXiv:0711.1243 
[17] Y. Q. Gu, The Vierbein Formalism and Energy-Momentum Tensor of Spinors, arXiv:gr-qc/0612106

[18] Y. Q. Gu, The Simplification of Spinor Connection and Classical Approximation, arXiv:gr-qc/0610001

[19] M. Mori, M. Umemura, Galactic evolutionary path from primeval irregulars to present-day ellipticals, Nature, 440, 644(2006), arXiv:astro-ph/0512424

[20] Y. Sofue, Y. Tutui, M. Honma, et al, Central Rotation Curves of Spiral Galaxies, arXiv:astro$\mathrm{ph} / 9905056$

[21] Y. Sofue, Central Rotation Curves of Galaxies, Astrophys. J., 458, 120(1996), astro-ph/0010595

[22] J. R. Bownstein, J. W. Moffat, Galaxy Rotation Curves without Non-Baryonic Dark Matter, Astrophys. J., 636,(2006), astro-ph/0506370

[23] Paolo Salucci, The mass distribution in Spiral galaxies, Proceedings IAU Symposium No. 244, 2007, arXiv:0707.4370

[24] M. Persic, P. Salucci, Rotation Curves of $96^{7}$ Spiral Glaxies, Astrophys. J., Suppl. v.99, p.501(1995), arXiv:astro-ph/9502091

[25] M. Persic, P. Salucci, F. Stel, The Universal Rotation Curve of Spiral Galaxies: I. the Dark Matter Connection, MNRAS, 281, 27(1996), arXiv:astro-ph/9506004

[26] L. M. Widrow, B. Pym, J. Dubinski, Dynamical Blueprints for Galaxies, Astrophys. J., 679(2), pp. 1239-1259(2008), arXiv:0801.3414

[27] Yu S. Y., Ho L. C., On the Connection Between Spiral Arm Pitch Angle and Galaxy Properties[J]. 2018, ApJ, 869, 29. 